\title{
Solar radiation - to - power generation models for one-axis tracking PV system with on-site measurements from Eskisehir, Turkey
}

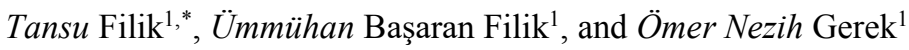 \\ ${ }^{1}$ Anadolu University, Faculty of Engineering, Eskisehir, 26555, Turkey
}

\begin{abstract}
In this study, new analytic models are proposed for mapping onsite global solar radiation values to electrical power output values in solar photovoltaic (PV) panels. The model extraction is achieved by simultaneously recording solar radiation and generated power from fixed and tracking panels, each with capacity of $3 \mathrm{~kW}$, in Eskisehir (Turkey) region. It is shown that the relation between the solar radiation and the corresponding electric power is not only nonlinear, but it also exhibits an interesting time-varying characteristic in the form of a hysteresis function. This observed radiation-to-power relation is, then, analytically modelled with three piece-wise function parts (corresponding to morning, noon and evening times), which is another novel contribution of this work. The model is determined for both fixed panels and panels with a tracking system. Especially the panel system with a dynamic tracker produces a harmonically richer (with higher values in general) characteristic, so higher order polynomial models are necessary for the construction of analytical solar radiation models. The presented models, characteristics of the hysteresis functions, and differences in the fixed versus solar-tracking panels are expected to provide valuable insight for further model based researches.
\end{abstract}

\section{Introduction}

The share of solar power generation inside the overall electricity generation is rapidly increasing, thanks to the developments in solar photovoltaic (PV) technology [1]. Solar PV systems convert the energy acquired from sun light (photons) into electricity (voltage), using the, so called, PV effect. Obviously, the total amount of average solar radiation value at a certain point is the most important indicator to determine the total electrical energy generation from the sun. This value naturally changes according to the geo-location of the panel, the time of the day (which determines the arrival angle of the sun lights), and the climatic conditions.

Modern electrical grid operations require an accurate estimation of power generation (including renewables, such as sunlight and wind). Non fuel-based power generation systems, such as solar PV panels, impose a challenging energy estimation problem because of the high uncertainty and statistical variation of the data $[2,3]$. In the literature, various

* Corresponding author: tansufilik@anadolu.edu.tr 
short-term forecasting models are proposed for wind and solar radiation. The mean hourly global solar radiation can usually be modelled analytically using geo-astronomical calculations and/or time series analysis of a well-structured solar radiation data $[4,5]$. These models can give the hourly global solar radiation values of an arbitrary region in the world. However, in order to accurately calculate the electricity output of the PV panels, other models are necessary. There are some models which depend on solar radiation on PV panels and ambient temperature [6]. These models discover the nonlinear relation between the solar radiation and the produced electrical energy.

In this study, we approach the modelling problem through simultaneous observation of solar radiation values, temperatures, solar power output of active sun tracking and fixed PV systems in a controlled environment in Eskisehir. A comparison of fixed and active solar tracking system on the same geo-location in Central Anatolia (Eskişehir) is also a novel contribution of this particular work. All these region specific parameters are recorded with very high resolution (below one minute intervals).

A critical observation is that the conversion of real averaged solar radiation values into real electrical power exhibits a hysteresis function relation for both the fixed and active tracking PV panels. The hysteresis is in the form that the nonlinear relation between a solar radiation value and its corresponding electrical power changes in time. This time variance was not reported or modelled in the literature before. As a next novel contribution, the nonlinear and time-varying relation between solar radiation and power generation is modelled as three-parts hysteresis function, corresponding to the morning, noon, and afternoon time segments. Finally, the third novel contribution of this work is achieved by performing the above analysis on both fixed and (single axis) active sun tracking systems. Therefore, efficient system models are expected to be achieved for two widely popular PV panel types.

\section{Experimental Setup}

Figure 1 shows an actual photo of the controlled experimental setup, where all the measurements are recorded. The experimental setup is inside the Anadolu University 2 Eylul Campus in Eskisehir region (39.78 latitude, 30.52 longitude, and $794 \mathrm{~m}$ altitude with respect to sea level). The installed solar energy generation system consists of 39 solar PV panels with $260 \mathrm{~W}$ capacity. The overall energy generation system is established as a hybrid renewable energy system which consists of both $6 \mathrm{~kW}$ on-grid $(3 \mathrm{~kW}$ fixed on the ground, $3 \mathrm{~kW}$ solar tracker) and $4 \mathrm{~kW}$ off-grid (roof mounted) solar panels combined with a $1 \mathrm{~kW}$ wind turbine. The active sun tracking system ads up to $3 \mathrm{~kW}$ through 12 panels with $260 \mathrm{~W}$ capacity, each. (as can be seen on the right hand side of Figure 1). Similarly, the fixed panel system also adds up to $3 \mathrm{~kW}$ with 12 panels of $260 \mathrm{~W}$ capacity (as can be seen on the left hand side of Figure 1). The recording station at the back is equipped with the necessary inverters, acquisition cards, computers and data storage units. Detailed technical specifications of the system can be reached from [7]. 


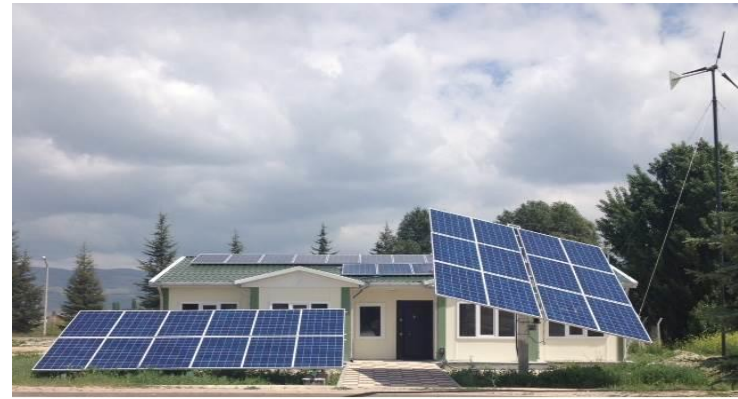

Fig 1. The Installed fixed (left side) and sun tracking solar PV panels (right side) with same capacity $(3 \mathrm{~kW})$ in Iki Eylul Campus of Anadolu University, Eskisehir, Turkey.

Figure 2 (a) shows the outdoor sensors which measure the solar radiation values, temperature values, wind speed / direction, humidity, pressure, etc., and Figure 2 (b) shows a photo of the real-time data recording system.

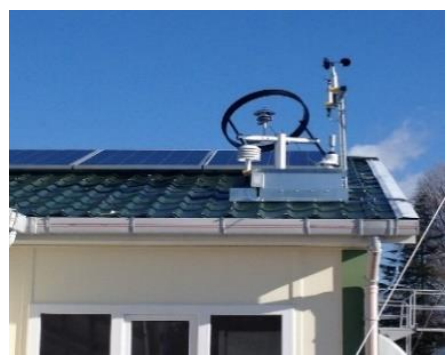

(a)

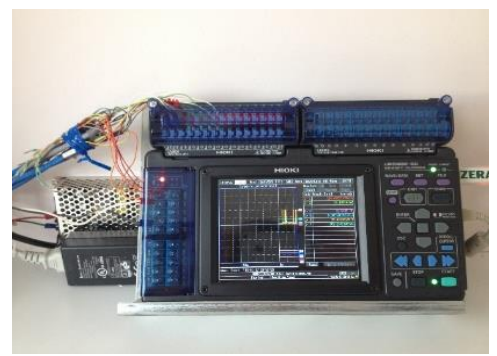

(b)

Fig 2. (a) Outdoor sensors, and (b) multichannel real time data recording system.

The generated electricity of active tracking and fixed PV panels are separately recorded on inverter with solar logging system which is shown in Figure 3. Also the global radiation values are instantaneously (with 10 seconds resolutions) recorded in data recording system for year 2016.

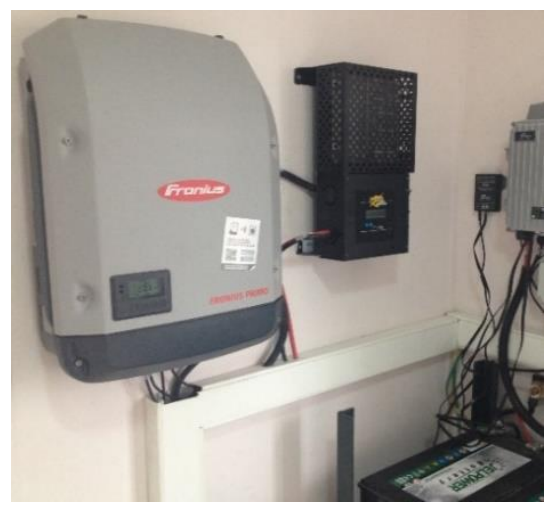

Fig 3. On-grid Inverter (Fronius) 


\section{Solar Radiation to Power Generation}

Classical models for the estimated conversion of solar radiation value into generated electricity power typically use average solar radiation, static values of PV parameters, and a fixed ambient temperature [6]. Practically, in order to roughly calculate the electrical potential of annual solar energy output from a PV system can be calculated as:

$$
E=A \cdot r \cdot G \cdot \rho
$$

where $A$ is the total solar panel area in $\mathrm{m}^{2}, r$ is the panel efficiency (yield) parameter, $G$ is the annual average solar radiation on tilted panels, and $\rho$ is the performance ratio of the overall system. This global formula naturally gives a rough annual electricity generation value, and it does not take into account instantaneous conversion values.

Solar radiation, however, is a dynamic process that depends on the geographic location and atmospheric conditions. The black-labelled curve $(g(t))$ in Figure 4 shows averaged global radiation values with the blue $\left(E_{f i x}(t)\right)$ and red $\left(E_{\text {trac }}(t)\right)$ curves corresponding to the instantaneous electrical powers generated from fixed and solar tracking PV panels, respectively. The plotted functions are averages of the daily measurements as a function of time over a 24-hour duration (starting from 00:00 at night and ending at 23:59). Using the same approach of Eq. 1, it can be shown that the instantaneous electricity output value for a PV panel can be calculated as:

$$
E(t)=A r(t) g(t) \rho(t),
$$

with the same parameters (only converted into functions of time) as in Eq. 1.

Unfortunately, the performance ratio and efficiency of the overall system also change with time (and temperature), as expected. Therefore, the need for an accurate model of $r(t)$ and $\rho(t)$ is necessary. In order to see the time varying behaviour of these parameters, a plot of real averaged electricity output values versus real averaged global radiation values of the active sun tracking and fixed panels are shown in Figure 5. Two very important observations can be made from the curves in Figure 5:

- The sun tracking system (in red) provides a higher conversion ratio than the fixed panel (this can be seen due to the red curves being above the blue curves).

- Sun rising (morning) conversion plots are different from the conversion curves of afternoon hours (exhibiting a hysteresis shape) for both the fixed and the sun tracking PV systems. In other words, a certain solar radiation value (say, $g$ ) causes the production of different electric power values depending on whether the value appears in the morning or in the afternoon.

It is, therefore, argued that there is a hysteresis phenomenon in the conversion function, probably due to the ambient temperature difference between morning and afternoon hours. The noon time is observed to constitute a threshold region. This hysteresis function means a time varying behaviour, therefore nonlinear models are required. In this study we propose a piecewise model (consisting of three-part functions) that considers morning, noon and afternoon hours separately. The proposed approach is used for the modelling of both fixed and solar tracking PV systems. 


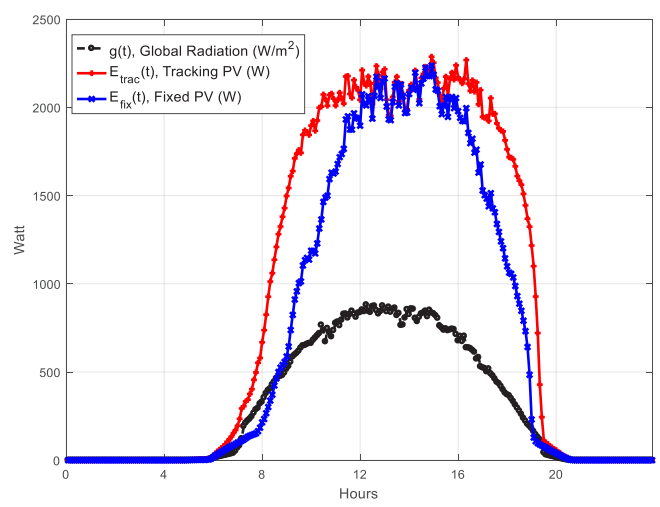

Fig. 4. Real averaged electricity generations of the installed tracking $\left(E_{t r a c}(t)\right)$ and fixed $\left(E_{f i x}(t)\right) \mathrm{PV}$ systems with the measured global radiation value $(g(t))$ between 1st July and 31st Dec., 2016.

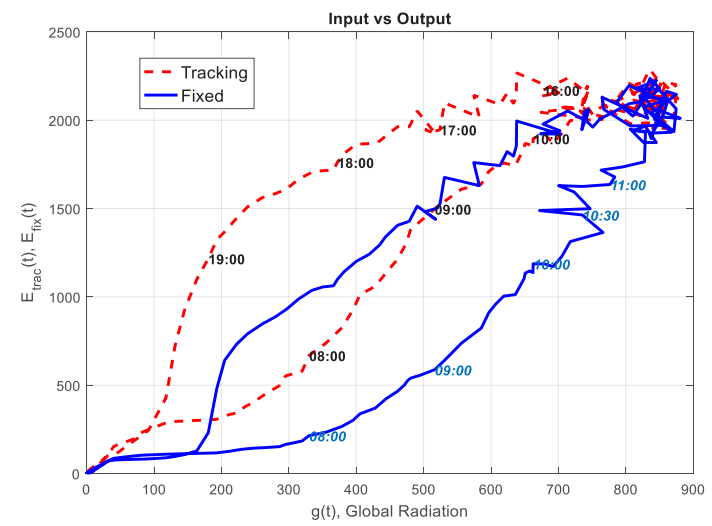

Fig. 5. Real averaged electricity output values versus real averaged global radiation values of the active sun tracking and fixed panels (blue for fixed panel, red for sun tracking system). Certain hour points are shown on the plots.

The proposed three-part functions for fixed and active sun tracking systems are as follows, respectively;

$$
\begin{gathered}
E_{\text {fix }}^{m}(t)=\left\{\begin{array}{lr}
a_{1}+b_{1} g(t)+c_{1} g^{2}(t), & 0<t \leq 11.5 \text { (morning) } \\
a_{2}+b_{2} g(t), & 11.5<t<15.4 \text { (noon/evening) } \\
a_{3}+b_{3} g(t)+c_{3} g^{2}(t), & 15.4<t \leq 0 \text { (evening/night) }
\end{array}\right. \\
E_{\text {trac }}^{m}(t)=\left\{\begin{array}{lc}
\alpha_{1}+\beta_{1} g(t)+\gamma_{1} g^{2}(t), & 0<t \leq 11 \text { (morning) } \\
\alpha_{2}+\beta_{2} g(t), & 11<t<17 \text { (noon/evening) } \\
\alpha_{3}+\beta_{3} g(t)+\gamma_{3} g^{2}(t), & 17<t \leq 0 \text { (evening/night) }
\end{array}\right.
\end{gathered}
$$

The coefficient parameters in these equations are estimated using minimum least square optimization from the recorded values between 1st July and 31st December, 2016. The least square optimized function coefficients for the records are presented in Table 1 for both the active sun tracking and fixed systems. The model generated energy generation values (over actual power values) are shown in Figure 6 (a). The fitted model conversion curves and real conversion curves are presented in Figure 6 (b). 
Table 1. Model (mapping) function coefficients.

\begin{tabular}{|c|c|c|c|c|c|c|}
\hline \multirow{2}{*}{ E(t) } & \multicolumn{3}{|c|}{ Fixed PV } & \multicolumn{3}{c|}{ Tracking PV } \\
\cline { 2 - 7 } & $\mathbf{a}$ & b & $\mathbf{c}$ & $\alpha$ & $\beta$ & $\gamma$ \\
\hline Morning & 12.515 & -0.433 & 0.003 & 1.233 & 1.844 & 0.0014 \\
\hline Noon/Evening & 1907.6 & 0.0002 & - & 2010 & 0.0002 & - \\
\hline Evening/Night & -18.439 & 3.093 & -0.0003 & -19.87 & 7.5741 & -0.0072 \\
\hline
\end{tabular}

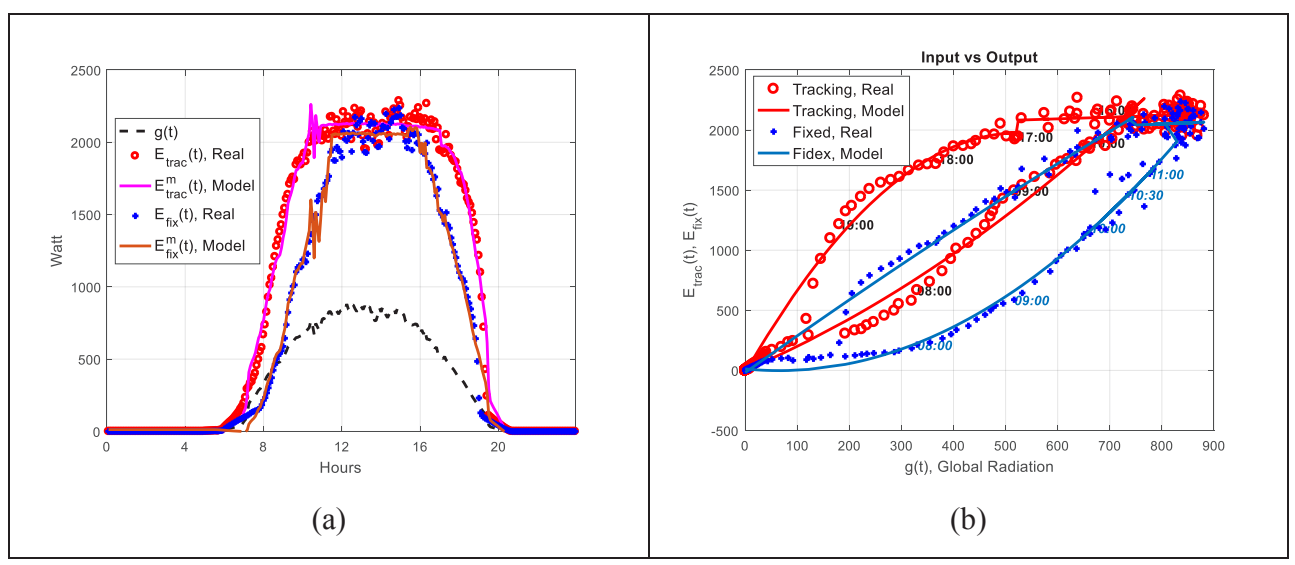

Fig. 6. Model vs. real values of (a) generated electric power, and (b) conversion functions.

Finally, real electricity generation and the estimated model electricity generation values are given in Table 2 for the remaining data set. It is shown that proposed model gives accurate results.

Table 2. Real electricity generation versus estimated model generation values.

\begin{tabular}{|c|c|c|}
\hline kWh & $\begin{array}{c}\text { Real } \\
\text { Generation }\end{array}$ & $\begin{array}{c}\text { Model } \\
\text { Generation }\end{array}$ \\
\hline Tracking PV & 26.362 & 26.516 \\
\hline Fixed & 20.672 & 20.834 \\
\hline
\end{tabular}

\section{Conclusion and discussion}

In this work, solar radiation and energy generation data obtained from our experimental set-up are used to illustrate various important aspects of the radiation-to-energy conversion phenomenon. First, it was observed that the conversion is not only non-linear, but it also has a strong time-varying characteristic. Particularly, the conversion rate is consistently higher in the afternoon hours as opposed to the morning hours, although the radiation value plot is necessarily symmetric around the noon time. This observation induced a novel hysteresis 
function modelling approach through separate piece-wise quadratic functions for the morning, noon, and afternoon. The resulting hysteresis model is found to achieve a small squared error when compared to the actual recordings.

The second important observation is that the efficiency gain of the sun tracking PV panels is clearly visible from the conversion curves. The curve for the sun tracking system is consistently tracing above the conversion values of the fixed panel.

The time varying hysteresis effect of the conversion curve is expected to be due to the ambient temperature and the operation characteristics of the PV materials. However, this correspondence needs to be examined and reported as a possible follow-up of this research.

This work was supported by Anadolu University Research Fund under contract no: $1505 F 512$

\section{References}

1. B. Parida, S. Iniyan, R. Goic, Renew. and Sust. Energy Reviews 15, 3, 1625-1636 (2011)

2. H. Farhangi, IEEE Power and Energy Mag. 8, 1 (2010)

3. P. Varaiya, F.W. Felix, J.W. Bialek, Proc. of the IEEE 99, 1, 4-57 (2011)

4. S.N. Kaplanis, Renew. Energy 31, 6, 781-790 (2006)

5. C. Gueymard, Solar Energy 68, 3, 285-303 (2000)

6. H. Yang, L. Lu, W. Zhou, Solar Energy 81, 1, 76-84 (2007)

7. T. Filik, U.B. Filik, Anadolu Univ. J. of Sci. and Tech. A-Appl. Sci. and Eng. 18, 1 (2017) 